\title{
Trinitarian pollen grain revealed by new technology
}

\author{
jingshi xue ${ }^{1}$, Li-jun Chen ${ }^{2}$, Zhong-Nan Yang ${ }^{1}$, and Wang Xin ${ }^{3}$ \\ ${ }^{1}$ Shanghai Normal University \\ ${ }^{2}$ National Orchid Conservation Center of China and Orchid Conservation \& Research \\ Center of Shenzhen \\ ${ }^{3}$ Chinese Academy of Sciences Nanjing Branch
}

July 20, 2020

\begin{abstract}
The origin of angiosperms has been a focus of botanical research. Although macrofossils and microfossils have suggested that angiosperms may have earlier occurrence, there are some scholars reluctant to accept these fossil records and their implications. To resolve this conflict studying more fossils using new technology is helpful. Obvious differences exist between pollen of seed plants and spore of ferns. Applying the MFCFM technology, here we report a half pollen grain with a mosaic combination of fluorescent spectral features of angiosperms and ferns from the Jurassic of China. Although the mother plant is still mysterious, the features of this fossil pollen grain apparently demands a new thinking on pollen and its evolution.
\end{abstract}

\begin{abstract}
:
The origin of angiosperms has been a focus of botanical research. Although macrofossils ${ }^{1,2}$ and microfossils ${ }^{3,4}$ have suggested that angiosperms may have earlier occurrence, there are some scholars reluctant to accept these fossil records and their implications ${ }^{5}$. To resolve this conflict studying more fossils using new technology is helpful. Obvious differences exist between pollen of seed plants and spore of ferns. Applying the MFCFM technology, here we report a half pollen grain with a mosaic combination of fluorescent spectral features of angiosperms and ferns from the Jurassic of China. Although the mother plant is still mysterious, the features of this fossil pollen grain apparently demands a new thinking on pollen and its evolution.
\end{abstract}

Keyword: ferns, angiosperm, pollen, fluorescence, spectrum

Pollen grains of seed plants can be easily distinguished from the spores of ferns in various aspects. Hitherto there is nothing intermediate between these two categories of microspores in terms of wall organization and fluorescence features, although such an intermediate, if existed, would provide otherwise unavailable meaningful implications for plant evolution. Here we report a half pollen grain from the Jurassic of Inner Mongolia, China. The MFCFM (Multiple Frequency Confocal Fluorescence Microscopy) ${ }^{6}$ reveals that 1) fluorescence spectrum of this pollen grain has two peaks, one resembling spore and the other resembling pollen; 2) its separated tectum and supratectal spines resemble certain angiosperms. Such an inter-class combination of features reveals a previously unknown aspect of plant evolution.

The fossil was collected from the outcrop of the Jiulongshan Formation (the Middle-Late Jurassic, $>164$ Ma) near Daohugou, Inner Mongolia, China (Fig. S1). The sample was removed from an unidentified fossil plant (Fig. S2a-c). Details of the pollen grain were observed and photographed using a Leo 1530 VP SEM and a Tescan MAIA3 SEM at the Nanjing Institute of Geology and Palaeontology. MFCFM observation was performed using Olympus FV3000 laser scanning microscope at the Shanghai Normal University. For comparison, Brassica juncea, Oryza sativa, Actinidia sinensis, Pinus kwangtungensis and Cryptomeria fortunei were selected as representatives of seed plants, andArachniodes, Ceratopteris and Lycopodium 
clavatum were selected as representatives of spore plants. Lycopodium clavatumwas procured from Sigma (19108-100G-F). Other pollen and spores were collected from the Botanical Garden of Shanghai Normal University for MFCFM. We used fluorescence with wavelength of $405 \mathrm{~nm}$ for excitation, and the bandwidth was set to $5 \mathrm{~nm}$. Fluorescence of total 53 frequencies ranging from 430 to $690 \mathrm{~nm}$ was used. To eliminate the noise from the background, the signal of the background (sediment matrix) was subtracted from that of the pollen grain. All photographs were saved in TIFF format and organized together for publication using a Photoshop 7.0.

The pollen was embedded in siltstone of volcanic ashes (Figs. S2a-c). By accident, the pollen grain was split in halves (Figs. 1a-b), and only one half was used in this analysis and the wall organization was observed using both SEM (Figs. 1a-d. g-h) and MFCFM (Figs. 1e-f). The pollen grain is close to round in outline, $22 \times 19 \mu \mathrm{m}$, uniaperturate. The wall includes a foot layer, columella, and separated tectum (Figs. 1a-h). The foot layer is present in both aperturate and non-aperturate regions, with smooth inner surface, slightly heterogeneous, $0.3-0.7 \mu \mathrm{m}$ thick (Figs. 1c-e,g). The columella is poorly developed only locally, ranging from nil to $1.25 \mu \mathrm{m}$ (Figs. 1b,g). The tectum is approximately $0.25 \mu \mathrm{m}$ thick, separated from the foot layer, with its inner surface visible in region where the neighboring foot layer is missing, well-separated from the foot layer in the margin of aperture, with supratectal spines (Figs. 1b-h). The foot layer and tectum are wellseparated in MFCFM image (Fig. 1e), similar to the pollen wall ofOryza sativa (Fig. 1i). The fluorescence spectrum of the pollen grain has two peaks, namely at $535 \mathrm{~nm}$ and $495 \mathrm{~nm}$, respectively, different from both spore of ferns and pollen of seed plants (Figs. 2a-b).

The fluorescence spectrum of the present pollen grain combines the features of both ferns' spore and spermatophytes' pollen. The observation on extant plant taxa indicates that fluorescence spectra of spores have their peaks in 510-545 nm, while those of pollen in 475-510 nm. As seen in Fig. 2, the fluorescence spectrum of the present pollen grain has two peaks, namely $535 \mathrm{~nm}$ and $495 \mathrm{~nm}$, respectively. One of two peaks matches that of ferns' spore and the other matches that of pollen in seed plants. This unique spectral feature is perplexing if consideration is restricted to extant plants. However, it becomes much easy to accept and understand if fossil plants are included consideration. There are fossil taxa termed as "seed ferns", which appear like ferns but are reproduced through seeds. Our new pollen seems to be of another taxon that appears weird, chimeric, spanning formerly isolated taxa in eyes of botanists who are more familiar to living taxa. Wide morphological and molecular gap among living taxa is conceivable because the intermediate ones have been deleted from the phylogeny tree. We assumed our fossil pollen will help to narrows the otherwise wide-remaining gap between ferns and spermatophytes.

Among the known spermatophytes, the present pollen grain appears more similar to certain angiosperms in wall organization. Interestingly, the well-separated tectum and foot layer seen in Fig. 1e are never seen in any gymnosperms, while frequently seen in some Poaceae (Oryza sativa) (Figs. 1i, S3), suggestive of possible angiosperm affinity. The supratectal spines on the tectum is another feature frequently seen in some angiosperms. Taking all into consideration, pollen wall organization of the present pollen grain favours that its mother plant is most likely an angiosperm, which we think is an open question requiring further investigation.

MFCFM oservation reveals that the Jurassic pollen grain reported here is unique in its fluorescence spectrum resembling microspores of both ferns and seed plants. The pollen wall organization demonstrates similarity to that of some Poaceae. The implications of this new fossil material need further exploration.

Acknowledgement We thank Mr. Yan Fang for his assistance with the SEM. This research was supported by the Strategic Priority Research Program (B) of Chinese Academy of Sciences (Grant No. XDB26000000), and National Natural Science Foundation of China (41688103).

\section{References}

$1 \mathrm{Fu}$, Q. et al. An unexpected noncarpellate epigynous flower from the Jurassic of China. eLife $\mathbf{7}$, e38827, (2018). 
2 Wang, X. The Dawn Angiosperms. Springer, Cham, Switzerland, 2018.

3 Hochuli, P. A. \& Feist-Burkhardt, S. A boreal early cradle of angiosperms? angiosperm-like pollen from the Middle Triassic of the Barents Sea (Norway). Journal of Micropalaeontology 23, 97-104, (2004).

4 Hochuli, P. A. \& Feist-Burkhardt, S. Angiosperm-like pollen andAfropollis from the Middle Triassic (Anisian) of the Germanic Basin (Northern Switzerland). Frontiers in Plant Science4, 344, (2013).

5 Herendeen, P. S., Friis, E. M., Pedersen, K. R. \& Crane, P. R. Palaeobotanical redux: revisiting the age of the angiosperms. Nature Plants 3 , 17015, (2017).

6 Xue, J.-S. et al. Phenylpropanoid derivatives are essential components of sporopollenin in vascular plants. Molecular Plant, (in porgress).

Figure captions

Fig. 1 A Jurassic half pollen grain and its details. c, columella; f, foot layer; t, tectum. A. A piece of rock fragment bearing the pollen grain (arrow). Bar $=0.1 \mathrm{~mm}$. B. Detailed view of the pollen grain marked by the black arrow in Fig. 1A. The triangle points to the aperture. Bar $=2 \mu \mathrm{m}$. C. Detailed view of the region marked by white arrow in Fig. 1B, showing the foot layer, columellae, and tectum. Bar $=1 \mu \mathrm{m}$. D. Wall organization of the pollen grain, marked by black arrow in Fig. 1B, showing the heterogeneous foot layer and tectum. Bar $=1 \mu \mathrm{m}$. E. MFCFM image of the pollen grain showing separated foot layer and tectum. The triangle points to the aperture. Bar $=5 \mu \mathrm{m}$. F. MFCFM image of the pollen grain showing supratectal spines (arrows) on the tectum. The triangle points to the aperture. Bar $=5 \mu \mathrm{m}$. G. Detailed view of the three-layered pollen wall structure (tectum, columella, and foot layer), marked with yellow arrow in Fig. 1B. Bar $=1 \mu \mathrm{m}$. H. Risen and separated tectum near the margin of aperture, marked by blue arrow in Fig. 1B. Bar $=1 \mu \mathrm{m}$. I. Separated tectum in the pollen wall of Oryza sativa. Bar $=5 \mu \mathrm{m}$. Fig. 2 Comparison among the fluorescence spectra of the fossil pollen grain and spore/pollen of extant plants. A. Comparison between the fossil pollen grain and spore of ferns. Note one of the spectrum peaks (left) is distinct from those of extant ferns. B. Comparison between the fossil pollen grain and pollen grains of extant seed plants. Note one of the spectrum peaks (right) is distinct from those of seed plants. Fig. S1. Geographical position of the fossil locality, Daohugou Village, Ningcheng, Inner Mongolia, China. The rectangular region in the inset map of Northeast China is shown in detail in the main map, in which the position of Daohugou village (close to the junction among Liaoning, Hebei, and Inner Mongolia) is marked by the arrow. Fig. S2 General information of the fossil. A. A plant embedded in siltstone of the Jiulongshan Formation (Middle-Upper Jurassic). Bar $=1 \mathrm{~cm}$. B. Detailed view of the area marked by arrow in Fig. S2a. Bar $=0.5 \mathrm{~mm}$. C. Detailed view of the area marked by arrow in Fig. S2b, the arrow points to the source area of sample shown in Fig. 1. Bar $=0.1 \mathrm{~mm}$. Fig. S3 TEM image showing a pollen grain of extant Oryza sativa(Poaceae) with separated foot layer and tectum. Bar $=1 \mu \mathrm{m}$. 


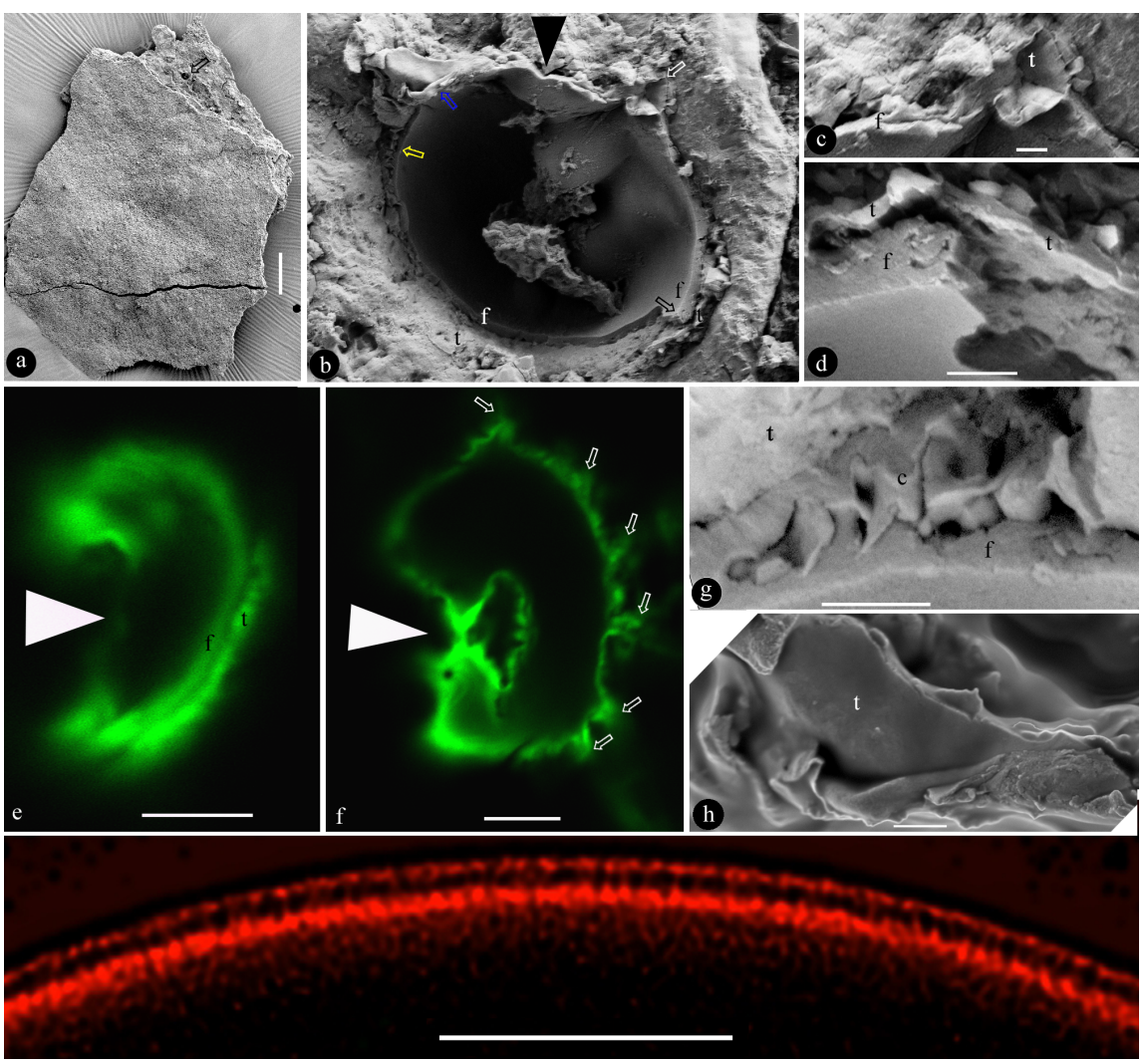



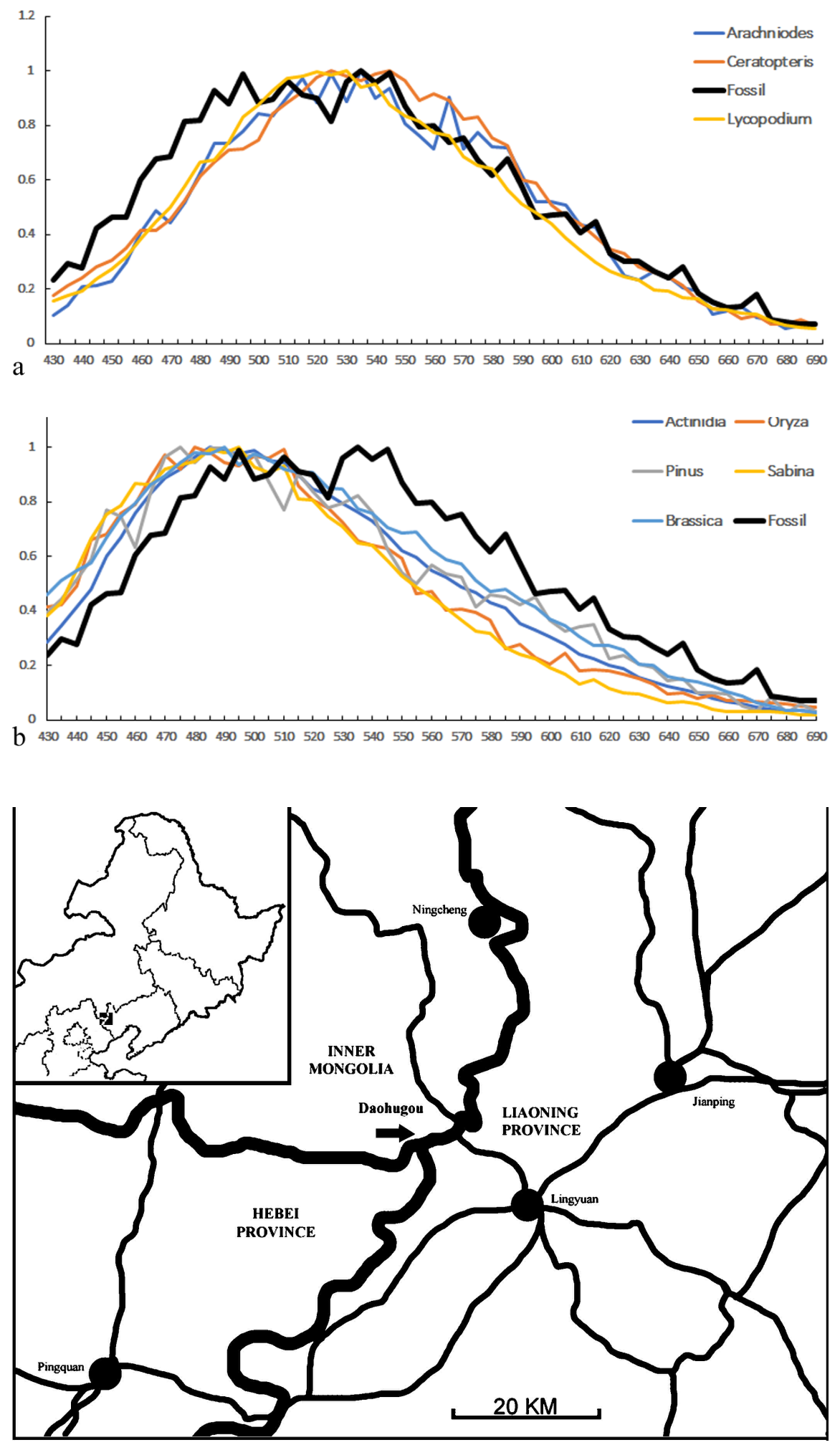

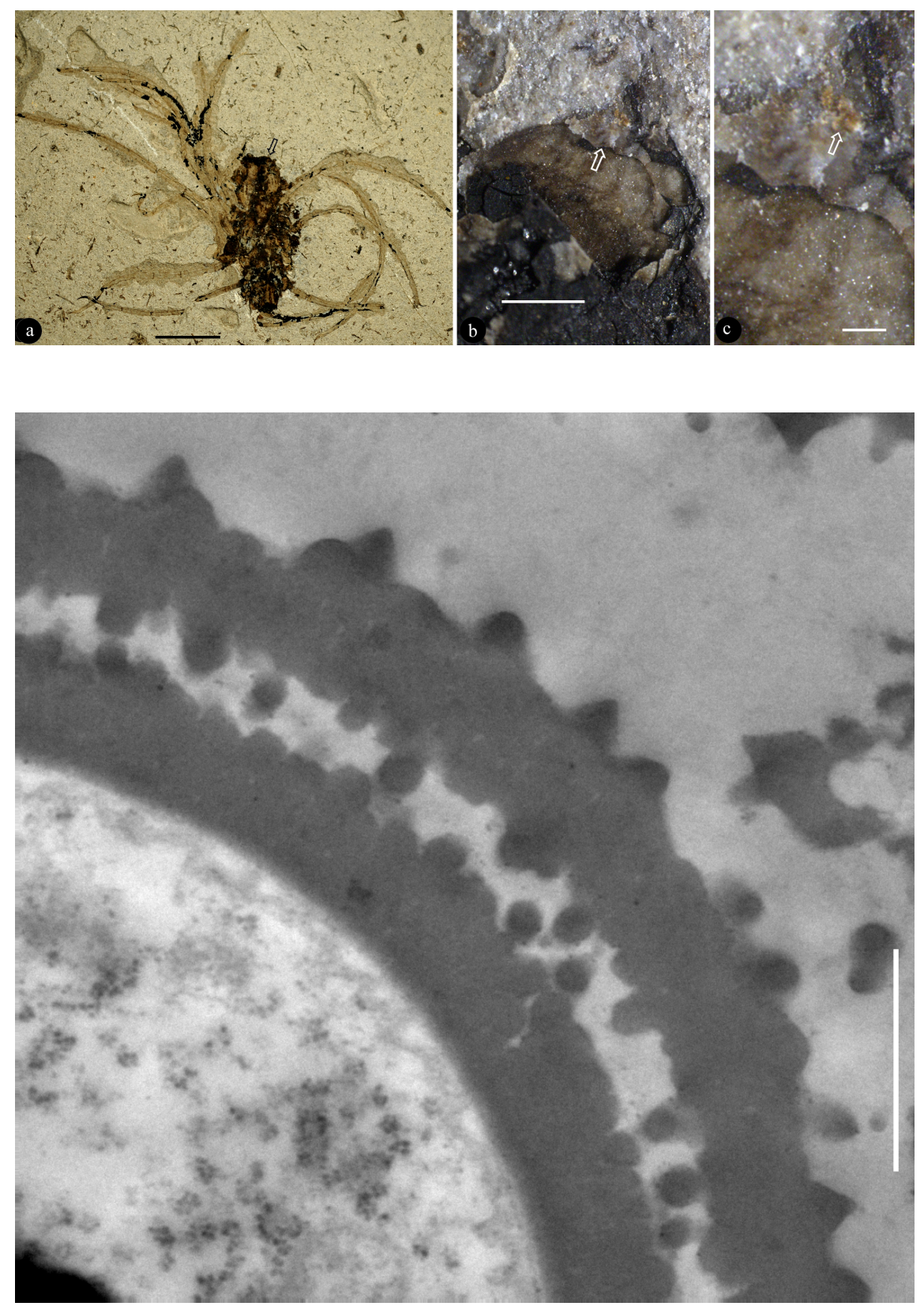